\title{
A sharp future for the 3.6-m DOT? The power of adaptive optics for medium size telescopes
}

\author{
Gilles Orban de Xivry ${ }^{1}$, Olivier Absil ${ }^{1}$, Vincent Moreau ${ }^{2}$ \\ ${ }^{1}$ Space sciences, Technologies, and Astrophysics Research (STAR) Institute, Université de Liège, \\ Allée du Six Août 19c, 4000 Sart-Tilman, Belgium \\ ${ }^{2}$ Advanced Mechanical and Optical System (AMOS), rue des Chasseurs Ardennais 2, \\ 4031 Angleur, Belgium
}

\begin{abstract}
Adaptive optics (AO), by compensating in real time the blurring due to atmospheric turbulence, improves the angular resolution and the sensitivity of ground-based telescopes. AO represents an opportunity for medium size telescopes to be competitive with respect to large and very large observatories. AO can be beneficial for most scientific cases and - we think - reachable with a reasonable effort. In this contribution, we review the current state of adaptive optics in medium size telescopes, discuss promising avenues and present a possible science case for the DOT. In the second part, we present the relevant expertise we are currently building up in Liège in the framework of the SALTO project, a 1-m telescope demonstrator with laser guided adaptive optics.
\end{abstract}

Keywords: adaptive optics - medium size telescopes - laser guide star - demonstrator - Devasthal Optical Telescope

\section{Introduction}

Adaptive optics ( $\mathrm{AO}$ ) compensates the atmospheric distortion of the wavefront and allows groundbased telescopes to achieve their theoretical diffraction limit. The AO correction depends strongly on wavelength, $\lambda$. Indeed the three fundamental parameters $r_{0}, \theta_{0}, \tau_{0}$ defining respectively the characteristics length, angle, and time ${ }^{1}$ of the atmospheric turbulence are all functions of $\lambda^{6 / 5}$. They underlie the core challenges and limitations of adaptive optics : 1) the spatio-temporal sampling imposed by those fundamental parameters scales with $\lambda^{-18 / 5}$, which makes adaptive optics much more difficult and challenging in the visible than in the near-infrared (NIR), 2) the corrected field-of-view with a single conjugated AO (using a single guide star) spans only a few arcseconds at optical wavelengths and a few tens of arcseconds in the NIR, 3) the required high spatio-temporal sampling and the small angular correlation impose the availability of a bright star in the near vicinity of the observed target to perform a good $\mathrm{AO}$ correction, thus limiting the sky coverage to at best a few percents in the NIR, and much less in the visible. Several concepts and new technologies have been developed to alleviate those limitations. Among them, the early introduction of laser beacons to create artificial sources

\footnotetext{
${ }^{1}$ Also referred as the Fried parameter $r_{0}$, the angular anisoplanatism $\theta_{0}$, and the time constant $\tau_{0}$ inversely proportional to the Greenwood frequency.
} 
has opened up, by extending the sky coverage, the use of AO to a larger community including extragalactic astronomy. Beyond artificial guide stars, a variety of concepts have since been devised and address particular science needs. Introduction to the field can be found in, e.g., Hardy (1998), Davies \& Kasper (2012).

Nowadays, AO is a scientific-driver technology in observational astronomy. Indeed, most large observatories have now at least one flavor of an AO system implemented in their ground-based telescopes. Nevertheless, high-resolution imaging remains limited due to the small number of large telescopes and the restrictions imposed by observing time and scheduling model. With the progresses in AO technologies, most of the key elements from fast wavefront sensor detectors to lasers and high-order deformable mirrors are now available commercially. Robust and affordable laser guided single conjugated AO system on medium size telescopes seems therefore accessible, giving an opportunity to upgrade the large number of 1-4 m existing telescopes. It provides medium size telescopes the opportunity for world-class science if they focus on scientific objectives that are hardly accessible on larger telescopes. A successful path initiated by Robo-AO, the autonomous laser guide star AO system (e.g. Baranec et al. 2018), relies on a robust, highly automated and easy to use system able to carry out follow-up observations of a significant fraction of the sky being observed by wide-field surveys such as GAIA (Gaia Collaboration et al. 2016), PLATO (Rauer et al. 2014), LSST (LSST Science Collaboration et al. 2009). Those surveys necessitate efficient follow-up to confirm and characterize the large number of discoveries made. The scientific topics range from transiting exoplanets and stellar systems to extragalactic objects such as lensed quasars. This route includes large population studies, rapid target characterization and long-term monitoring (Baranec et al. 2017).

In this paper, we review the current state of adaptive optics systems on medium size telescopes, in particular those assisted by laser guide stars (LGS). We present different avenues that we consider to be the most promising : from automated adaptive optics like Robo-AO to ground-layer adaptive optics. We elaborate a possible science case for a single conjugated LGS-AO with appropriate instrumentation : the follow-up of GAIA strongly-lensed quasars (Section 2.1.1). In Section 3, we present the Belgian project SALTO (Smart Adaptive optics and Laser guide star for medium Telescopes and Optical communications) : a 1-m class demonstrator of LGS-AO that will help us benchmark robust AO systems for 1-4 m telescopes as well as to test innovative concepts. We provide a system overview and present the expected performances. Finally, we discuss the possibilities of a sharp future for the DOT in the light of the current and under-construction instrumentation.

\section{Adaptive optics on medium size telescopes}

Adaptive optics in astronomy started in the 90's on 2-4 m telescopes (see e.g. Hardy 1998). The early work was done in the red visible and rapidly moved to the NIR thanks to the higher performances in that wavelength range and the complementarity with HST (e.g. Close et al. 2018). Nowadays, most AO system developments are done for the current 10-m class telescopes and are more and more focussed towards the coming Extremely Large Telescopes (ELTs).

Yet, there are currently a few medium size telescopes providing AO-assisted scientific observations. The current LGS AO scientific facilities are - to the best of our knowledge -: the Shane AO at Lick (3-m), Robo-AO at the University of Hawaii (UH) 2.2-m and SOAR Adaptive Module (SAM) at the 4.1-m Southern Astrophysical Research Telescope (SOAR), each implementing different concepts. The Shane AO at Lick can be operated as a natural guide star (NGS) or Sodium LGS system. It uses two deformable mirrors in a woofer-tweeter configuration providing a very good correction in the NIR and a partial correction in the red-visible $(0.7-2.2 \mu \mathrm{m})$. It is in operation since 2014 for scientific uses and provides its high Strehl to an imager, spectrograph, coronagraph and polarimeter (Gavel et al. 2014). Robo-AO uses a UV laser and focusses on automatization. The system has moved 
to several telescopes and is now installed on the UH 2.2-m. It comes with an imager and improves the angular resolution and sensitivity in the visible $(0.4-1 \mu \mathrm{m})$. The system is being upgraded (RoboAO2) to feature a better LGS wavefront sensor (WFS) detector, and will include a complementary low-noise NGS WFS. Together with the superior site of Mauna Kea, this will boost the performance of the system, delivering diffraction-limited images to an unprecedented number over a large spectral range and with rapid response time to high-priority events (Baranec et al. 2018). SAM at SOAR also uses a UV laser focussed at low altitude at $\sim 7 \mathrm{~km}$ (Tokovinin et al. 2016). Thanks to this configuration, SAM corrects essentially the ground layer. This ground-layer AO (GLAO) compensation is a partial correction but uniform over a wide field-of-view. Therefore, it provides a modest resolution $\left(0.3^{\prime \prime}\right.$ to $0.5^{\prime \prime}$ - corresponding to the free-atmosphere seeing) going up to the visible ( $I$-band) and on a large field-of-view ( $\left.3 \operatorname{arcmin}^{2}\right)$. SAM is in regular science operation since 2014.

NGS-AO systems are undoubtedly more common with among them a few innovative systems, like AOLI on the 4.2-m William Herschel Telescope (WHT), 'Imaka on the UH 2.2-m, and the planned AO system for the Eastern Anatolia Observatory (DAG 4-m). AOLI combines a low-order non-linear curvature wavefront sensor with Lucky Imaging (e.g. Mackay et al. 2012). It performs a modest correction but only needs a relatively dim star to perform the wavefront sensing (limiting magnitude $I \sim 16.5-17.5 \mathrm{mag}$ ). Thus thanks to its WFS it has a much wider sky coverage than a classical NGS SH-WFS. Under good conditions, it can also provide a good correction in the visible thanks to the Lucky Imaging. 'Imaka on the UH is a NGS-based GLAO demonstrator on Mauna Kea feeding a very wide-field imager $\left(10^{\prime} \times 10^{\prime}\right)$. It is distinct from a classical AO system and should be thought as a wide-field "super-seeing" system (Abdurrahman et al. 2018). The DAG-AO is the planned AO system for the DAG 4-m telescope. It is based on a pyramid wavefront sensor using a natural guide star. It aims to be a flexible AO : an extreme AO (ExAO) system in good conditions (good seeing and bright star) and a classical AO in less favorable conditions. The design is based on state-of-the-art technologies and concept: a high actuator density deformable mirror, a pyramid WFS, and a low-noise electron-multiplying CCD (EM CCD) WFS camera (Jolissaint et al 2018).

\subsection{Single conjugated and automated adaptive optics}

The forthcoming decades of astronomy will be dominated by wide-field surveys, such as GAIA, PLATO, or LSST. These large-sky surveys require characterizing thousands of discoveries with the help of high-resolution and deep images, spectroscopic observations, as well as time-domain astronomy. As suggested by Baranec et al. (2017), these science objectives can be classified into three major categories : large population studies, rapid target characterization, and long-term monitoring.

For many science goals, only a very efficient (in terms of observing time) LGS AO-assisted instrument, such as Robo-AO, can confirm and characterize the properties of a significant fraction of those large surveys. First installed on the 1.5-m at Palomar, then on the 2-m Kitt Peak, and now on the 2.2-m UH, Robo-AO has dominated the host star follow-up field, performing the follow-up of several thousands of Kepler Object of Interests (e.g. Law et al. 2014, Baranec et al. 2014, Ziegler et al. 2016). The automatization and minimal overhead times have enabled Robo-AO to typically complete 200-250 observations per night with a spatial resolution of $\sim 0.2^{\prime \prime}$ in the red-visible domain.

Considering the coming wide-field surveys, there is an increased need for such automated AO facilities. For example PLATO, which is devoted to the detection and characterization of terrestrial exoplanets in the habitable zone of solar-type stars, is highly dependent on various ground-based follow-ups. Due to its coarse plate scale, expected to be about $15^{\prime \prime} /$ pixel, a high-resolution follow-up needs to 1 ) discard false detections (estimated to be $\sim 15 \%$ of PLATO candidates) before more time is spent to perform spectroscopic measurements, 2) search for secondary sources that dilute the transit signal, thus reducing the measured planet radius, 3) provide statistics on the rate of planet occurrence 
as a function of host star multiplicity. Those objectives would be best addressed by a natural guide star single conjugated AO system, highly efficient and providing a correction up to $650 \mathrm{~nm}$. But this is only one example among several. In Section 2.1.1 we detail another attractive science case which consists in performing the follow-up of GAIA strongly-lensed quasars.

\subsubsection{A science case : follow-up of GAIA strongly-lensed quasars}

Despite not being dedicated to extragalactic survey, GAIA will also produce the first exhaustive allsky survey of QSOs with high-angular resolution. The survey is expected to be complete down to a G-mag $\approx 20$. The final catalog is expected for 2020 and the first data released was published in September 2016. While GAIA can attain an angular resolution of $\sim 0.2^{\prime \prime}$, the first data release (DR1) has been limited to $2^{\prime \prime}-4^{\prime \prime}$. The second data release (DR2, 2018) now reaches in the best case $\sim 0.4^{\prime \prime}$ with completeness for separations larger than $\sim 2.2^{\prime \prime}$ (Gaia Collaboration et al. 2018, Krone-Martins et al. 2018). Ultimately, as the resolution improves in future DR and methods to mine gravitational lenses are refined, GAIA will provide an all-sky magnitude-limited census of strongly-lensed quasars down to image separations as small as $\sim 0.18^{\prime \prime}$ (e.g. Krone-Martins et al. 2018).

Currently, a total of about 200 lensed quasars are known, with a median image separation of $1.8^{\prime \prime}$ (see the online data base ${ }^{2}$ compiled by Lemon et al. 2018). Finet $\&$ Surdej (2016) have shown that out of the expected $>5 \times 10^{5}$ QSO detections by GAIA, about 3000 may be multiply imaged lensed quasars, with about 250 systems consisting of more than two lensed images. This $\sim 10$-fold increase is mostly the results of the enhanced spatial resolution allowing to resolve lenses with small image separations. Interestingly this will also probe different galaxy populations of deflectors (more latetype systems) and higher redshift than any other surveys. Based on GAIA DR2, a few discoveries have already been made. Recently Lemon et al. (2018) confirmed the discovery of 22 new gravitational lensed quasars (with 3 quadruples among them) and Krone-Martins et al. (2018) discovered two new quadruply-imaged quasars. Finally, even in the LSST era we can expect the GAIA sample to remain unique due to its small angular separation completeness unreachable by seeing-limited ground-based instrumentation.

In order to prove the lensing nature of these candidates and to exploit them scientifically, highangular resolution in multiple bands and spectroscopic observations are required. The scientific applications are numerous and we list a few hereafter (e.g. see Rusu et al. 2016 for high-resolution imaging applications) : 1) confirming the lenses through the acquisition of redshifts of the multi-images of the quasars, 2) modeling of the lensed quasars and deflectors and the subsequent analysis of the distribution of dark matter in those deflectors, 3) studying the quasar galaxy hosts and the correlation with the black hole mass (e.g. $M_{B H}-\sigma_{*}$ ), 4) using microlensing to probe e.g. the accretion disc (e.g. Sluse 2019), 5) measurement of the Hubble constant using time-delay measurements between the multiple images. All those applications require ground-based AO-assisted follow-up either imaging or spectroscopy and often long-term monitoring. The backend instrument would cover visible and NIR wavebands in order to observe a maximum of emission line features of QSOs - within the capabilities of the AO system. Ideally, the follow-up would be done by a medium-resolution integral field unit (IFU) spectrograph $(R \sim 2000)$ with a field-of-view of a few arcseconds. Alternatively, a high-resolution imager performing multi-band imaging would already provide a wealth of information. Together with the automatization, the key challenge for such facility would be to offer the largest sky coverage in order to be able to follow-up a substantial fraction of the $\sim 3000$ GAIA lenses. From a first coarse analysis, a single Rayleigh laser guide star focussed at $\sim 16 \mathrm{~km}$ altitude could already provide a sky coverage of $\sim 50 \%$ for a minimum Strehl of $\sim 25 \%$ in the K-band. Alternatively, a LGS-GLAO system with one or several guide stars could provide a modest correction up to the

\footnotetext{
${ }^{2}$ https://www.ast.cam.ac.uk/ioa/reasearch/lensedquasars
} 
Table 1: Taxonomy and qualitative comparison of the different AO concepts. See also e.g. Rigaut \& Neichel 2018 and Davies \& Kasper 2012.

\begin{tabular}{|l|lll|}
\hline Type & Description & Level of correction & Field-of-view \\
\hline SCAO & Single conjugated AO with a NGS & very good & a few 10's of arcsec \\
LGS-AO & SCAO with a LGS & medium & a few 10's of arcsec \\
ExAO & Extreme Adaptive Optics (NGS) & excellent & a few arcsec \\
GLAO & Ground Layer AO (with NGS or with LGS) & low & up to 10 arcmin \\
LTAO & Laser Tomography AO & good & a few 10's of arcsec \\
MCAO & Multi-Conjugated AO & good & up to 2 arcmin \\
MOAO & Multi-Object AO & very good & several arcmin \\
\hline
\end{tabular}

red-visible wavelength and cover a larger sky coverage.

\subsection{Beyond a conventional adaptive optics system}

NGS and LGS SCAO are by design limited in field-of-view and in their level of correction. Overcoming those limitations means a higher level of complexity anti-correlated with the amount of compromise one is ready to make. Beyond the single conjugated AO system with natural or laser guide star, there is a large taxonomy of AO systems. We refer to review articles for an introduction to those different AO species (e.g. Rigaut \& Neichel 2018 and Davies \& Kasper 2012). To give an idea of the different concepts, we provide a qualitative comparison in Table 1. In the following, we discuss two interesting avenues that have arguably a great potential for 4-m class telescopes despite a higher level of complexity.

Ground layer adaptive optics. GLAO compensates low altitude turbulence and can be seen as a trade off between compensation quality against field-of-view and uniformity. The concept is motivated by the presence of a dominant ground layer at most astronomical sites and the dome turbulences that are both major contributors to the total wavefront perturbations (e.g. Tokovinin et al. 2003, Egner et al. 2007). In parallel a large set of science applications can benefit from the image enhancement provided by GLAO and do not require diffraction limit. In fact, nearly all seeing-limited science cases would benefit from a gain of a factor $\sim 2$ in spatial resolution (in the NIR) as delivered by a GLAO system.

By measuring the ground-layer turbulence and using a single deformable mirror, GLAO offers a wide-field correction ranging from approximately $2 \operatorname{arcmin}^{2}$ to $10 \operatorname{arcmin}^{2}$. When assisted by laser guide stars, it also provides nearly full sky coverage because of the modest requirements on tip-tilt correction. The advantages of GLAO are : the increased point source sensitivity, the increased slit coupling efficiency for spectroscopy, the reduced crowding noise in dense fields, and the improved spatial resolution. In addition, this "seeing enhancer" delivers high image quality more frequently and more stably over time. It also provides a well-behaved PSF that is essentially uniform over the field-of-view. Finally, some improvements have been observed up to the red visible (Tokovinin et al. 2016, Abdurrahman et al. 2018). Science topics range from imaging to multi-object spectroscopy and from the nearby to the extragalactic universe. It allows the detailed study of nearby galaxies and their nuclear star cluster (Georgiev et al. 2019). It can also provide high spatial and spectral resolution of distant lensed quasars (e.g. Rabien et al. 2019, Fan et al. 2019) and partially resolve distant galaxies (typically of order $0.3^{\prime \prime}$ to $0.6^{\prime \prime}$ ). 
A few demonstrators have proven the concept of GLAO both with NGS or LGS, e.g. MAD (Marchetti et al. 2003) and MMT (Hart et al. 2010). Current facilities are SAM at SOAR implementing a single LGS-GLAO, and 'Imaka that demonstrates the potential of GLAO at Mauna Kea by implementing a wide-field NGS-GLAO $\left(>10^{\prime} \times 10^{\prime}\right)$ on the UH 2.2-m. On 8-m class telescopes, ARGOS, the multiple laser guide stars and wavefront sensing facility for the LBT, delivers now routinely GLAO correction greatly enhancing the science delivered by the NIR multi-object spectro-imager LUCI (Rabien et al. 2019). With the additional success of GRAAL at VLT, there is a growing interest for GLAO systems and new projects are currently initiated e.g. the ULTIMATE at SUBARU (Rigaut et al. 2018), for the 12-m Chinese LOT telescope (Jia et al. 2018) or for Keck (Lu et al. 2018).

Visible adaptive optics. Visible-AO is not an AO concept as those listed in Table 1, but comprises any attempt to use $\mathrm{AO}$ at visible wavelengths. In fact all the concepts in Table 1 can actually be appropriate depending on the scientific need. Until recently, NIR-AO has been favored for the complementarity offered to HST in visible light resolutions. But with the progressive HST end of life, visible-AO is regaining the favor of the astronomical community. The reasons it has not been more important are likely 1) the low Strehl of those first generation systems, and 2) all the visible photons in the case of NGS-AO were used by the wavefront sensor (Close et al. 2018). The recent advances in hardware, such as EM CCD visible fast detectors, NIR avalanche photodiode detectors, or deformable mirrors technologies, allow improved performances and alleviate the challenges associated with visible-AO. There are now a couple of systems able to provide reasonably good correction up to visible wavelengths, e.g. MagAO on the 6.5-m MMT, the ESO Adaptive Optics Facility (AOF) system with MUSE, or the SCExAO system with e.g. VAMPIRES (Norris et al. 2015). The scientific interest and the maturity of those second generation AO systems has lead to a rapid increase of scientific papers using visible-AO systems (Close et al. 2018).

In addition to be challenging, high Strehl visible-AO systems provide so far only a narrow fieldof-view and require a bright object to perform the correction. E.g. MUSE with the AOF provides a Strehl $>10 \%$ in the wavelength range of 465-930 nm over a field-of-view (FoV) of $7.5 \operatorname{arcsec}^{2}$. Going beyond the field-of-view limitation in the visible means performing multi-conjugated adaptive optics. This is what is proposed with MAVIS ${ }^{3}$ for the ESO's VLT AOF. This ambitious project aims at providing a $30^{\prime \prime}$ FoV close to the diffraction limit (15-20 mas Full Width at Half Maximum, FWHM) in the $450-980 \mathrm{~nm}$ and a large sky coverage of $>30 \%$. This pathfinder will undoubtedly open the road to a wider access to visible-AO astronomy.

On a 3.6-m telescope, we can expect $<40$ mas resolution in the $R$-band, better than the resolution of a $10-\mathrm{m}$ telescope in the $K$-band. AO correction in the visible on a 4-m telescope can take several forms, e.g. an extreme AO system based on a bright NGS, or a LGS-AO with a Sodium, or - as the results from 'Imaka suggest for Mauna Kea - if only modest improvement is sufficient, a GLAO system based on one or several Rayleigh laser guide stars.

\section{SALTO: the Belgian adaptive optics demonstrator}

In the framework of the SALTO project, we intend to build a 1-m class demonstrator with a laser guide star AO. The project objective is to develop our expertise and benchmark robust AO systems for 1-4 $\mathrm{m}$ telescopes. The end goal is to be able to offer affordable adaptive optics systems that are reliable and relatively easy-to-use in order to improve the complementarity of small and medium versus large telescope apertures. Besides astronomy, small-size telescopes assisted by LGS AO are also of interest for space debris tracking and imaging, and ground-space optical telecommunications. In the following

\footnotetext{
${ }^{3}$ http://mavis-ao.org/mavis/
} 
sections, we describe the concept, review the key preliminary design of the AO system and shortly discuss the expected performances. Part of the following material has also been presented in Orban de Xivry et al. (2018).

\subsection{System overview}

The SALTO project is a complete AO demonstrator including the telescope, the laser and the AO systems. It will be installed in the Belgian countryside allowing a fast track installation and commissioning. The site being a poor location for astronomy standards, it requires a certain complexity of the AO system. Therefore, despite its small size, the large seeing (expected to be in the 2-3" range) imposes a relatively high degree of correction similar to the one needed on 2-4 $\mathrm{m}$ telescopes on astronomical sites.

A first part of the project is to measure the strength of the atmospheric turbulence and elaborate forecasting tools. On one hand, we are starting a differential image motion monitor (DIMM) campaign to measure the integrated seeing. This elemental measurement helps us to tailor the AO design to the actual atmospheric conditions. On the other hand, we are setting up atmospheric turbulence forecasting tools. Based on mesoscale inputs and appropriate forecasting model, we will estimate the atmospheric conditions including the $C_{n}^{2}$ profiles. These will be compared in retrospective to our integrated seeing measurements.

Beside site testing and weather forecast, there are a number of developments we are carrying out in the framework of this project. These encompass all aspects of a small AO facility:

Prediction of performance through error budgets and end-to-end simulations. This is the first step required to determine the system parameters, predict the performance of the system, and study the sensitivity of the system to particular effects. See also Section 3.3.

Telescope design. Several designs are traded-off to optimize the optical quality, the available fieldof-view, the easiness of alignment, and cost. The telescope diameter will be $700 \mathrm{~mm}$.

Laser launch design. The laser system is based on a green $532 \mathrm{~nm}$ Rayleigh LGS focussed at about $10 \mathrm{~km}$. A short discussion and trade off can be found in Orban de Xivry et al. (2018). The laser itself should provide $15-20 \mathrm{~W}$ power at a pulse repetition rate of about $10 \mathrm{kHz}$. The current optical design of the launch system is refractive and uses a large aspherical lens at the exit, aiming for an intrinsic spot size of about $1^{\prime \prime}$.

Adaptive optics design. The system is a single conjugated AO system based on a Shack-Hartmann wavefront sensor. The system includes a NIR imager used to perform the natural guide star tip-tilt sensing and to analyze the performance of the system (Strehl ratio, FWHM, or encircled energy). The system is shortly described in Section 3.2.

Real-time control. The real-time computer must perform the data calibration, the wavefront gradient computation, the modal reconstruction and finally controls the deformable mirror to perform the phase correction. The delay between the frame acquisition and the control of the deformable mirror (DM) should be minimum (on the order of the milli-second) to ensure a proper rejection of the atmospheric turbulence. For SALTO, we will favor a CPU system and we will base the real-time computer software on open-access development such as DARC ${ }^{4}$ or $\mathrm{CACAO}^{5}$.

\footnotetext{
${ }^{4}$ Durham Adaptive Optics Real-time Controller, https://github.com/agb32/darc

${ }^{5}$ Compute And Control for Adaptive Optics, https://github.com/cacao-org/cacao
} 


\subsection{AO module preliminary design}

The AO and imaging systems will sit on the Nasmyth focus of the demonstrator, providing a gravity gradient-free platform. The optical layout follows a classical scheme : after a common deformable mirror correcting tip-tilt and higher-order aberrations (about 70 modes), the light is split between the laser light $(532 \mathrm{~nm})$ going to the wavefront sensor, and the red-visible and NIR light going to the imager. For the WFS, we will implement a gated Shack-Hartmann with $10 \times 10$ subapertures, each with $6 \times 6$ pixels. The gating is synchronized with the laser firing to expose the detector to the backscattered light coming from $10 \mathrm{~km} \pm 300 \mathrm{~m}$. The laser will be stabilized on the WFS by controlling a tip-tilt mirror in the laser launch system. The natural tip-tilt will be sensed by the imager detector and controlled by the deformable mirror. The selected key subsystems are summarized in Table 2.

Table 2: Selected key subsystems.

\begin{tabular}{|ll|}
\hline Subsystem & Values [nm rms] \\
\hline Deformable mirror & single DM with 97 actuators \\
& $\sim 70$ modes controlled \\
LGS wavefront sensor & $10 \times 10$ Shack-Hartmann \\
detector & EM CCD $128 \times 128$ \\
subaperture & $6 \times 6$ pixels \\
Max. framerate & $1 \mathrm{kHz}$ full frame \\
& $1.8 \mathrm{kHz}$ in $64 \times 64$ \\
Tip-tilt sensor and imager & InGaAs detector \\
Max. framerate & $600 \mathrm{~Hz}$ framerate full frame \\
\hline
\end{tabular}

\subsection{Predicted performances}

An example of basic error budget is given in Table 3. It assumes a $2^{\prime \prime}$ seeing $\left(r_{0}=5 \mathrm{~cm}\right)$ following a standard altitude distribution (Hufnagel-Valley profile). The total higher order wavefront rms error amounts to $\sim 146 \mathrm{~nm}$, or a Strehl of $\sim 15 \%$ in the R-band and $\sim 70 \%$ in the H-band.

Table 3: High-order error budget.

\begin{tabular}{|lr|}
\hline Error source & Values [nm rms] \\
\hline Focus anisoplanatism & 82 \\
Fitting error & 62 \\
Aliasing error & 36 \\
Temporal error & 91 \\
Wavefront measurement error & 33 \\
Multispectral error (zenith) & 0 \\
Total & 146 \\
\hline
\end{tabular}

End-to-end simulations allow us to refine this picture by including several effects such as e.g., the telescope pupil, realistic influence functions for the deformable mirror, the wavefront sensor detector properties, the laser uplink propagation, and scintillation. Because the turbulence will be relatively strong $\left(r_{0}=5 \mathrm{~cm}\right.$ and below) and our spatial sampling is relatively high $(d=7 \mathrm{~cm})$, we expect the flux within our WFS subapertures to vary strongly over time due to scintillation. We perform Fresnel 
propagation between the layers of our turbulence model and reproduce the expected phase and amplitude aberrations. The simulated flux standard deviation between subapertures is $\sim 50 \%$. This strong fluctuation has required further investigation to ensure the loop stability and decent performance. Including those different effects, we expect a total of about $160 \mathrm{~nm}$ rms wavefront error. This is slightly higher but consistent with our initial error budget. Using a different metric, this should represent a decrease of a factor $>4$ in FWHM at $658 \mathrm{~nm}$ providing a diffraction limited PSF (Point Spread Function) with $<0.4^{\prime \prime}$ in FWHM.

\section{Discussion}

A sharp future for the DOT appears to be a natural avenue for the telescope to boost its impact and increase its complementarity with larger telescopes. The important question, from a scientific and engineering point of view, is which adaptive optics system is best suited and achievable within a foreseeable future. This question is intimately linked to the backend instrument(s) the AO should serve.

There are several instruments currently available or under construction : the $4 \mathrm{k} x$ 4k CCD optical imager (Pandey et al. 2016), TIRCAM2 the NIR imager (Baug et al. 2018), ADFOSC the visible spectro-imager and polarimeter (Omar et al. 2019), TANSPEC the NIR spectro-imager (Ojha et al. 2018), and DOTIFS the multi-object integral field spectrograph (Chung et al. 2014).

The visible instruments: the CCD optical imager, ADFOSC and DOTIFS are unlikely to take full benefit of an AO system considering the short wavelengths, their wide field-of-view and their seeinglimited pixel scale (from $\sim 0.1$ to $0.8^{\prime \prime} /$ pixel). In the NIR, TIRCAM2 has a modest field-of-view (1.44 $\left.\operatorname{arcmin}^{2}\right)$ and a seeing-limited pixel scale $\left(\sim 0.17^{\prime \prime} /\right.$ pixel). TANSPEC on the other hand provides a Nyquist sampling at the diffraction limit, has a modest field-of-view $\left(1 \operatorname{arcmin}^{2}\right)$ and covers the NIR. A GLAO system would be an interesting avenue but while the current instrumentation would be able to demonstrate its benefits (depending on the strength of the ground layer), no current instrument seems to be ideal to take full advantage of the modest correction over a wide field-of-view (approx. $2^{\prime}$ to $10^{\prime}$ ). On the other hand, TANSPEC assisted with an LGS-AO system would open up high-resolution imaging and spectroscopy for the DOT. It would be able, e.g. to address partially the follow-up of strongly-lensed quasars of GAIA. Finally, the on-going development of SALTO will provide all the tools and expertise for a rapid implementation of such AO on the DOT.

\section{Acknowledgements}

The authors acknowledge the support from the Walloon region of Belgium through the program Skywin (project SALTO). GOX acknowledges support from the Beware Fellowships Academia program (convention \#1610368). OA acknowledges funding from the Fonds de la recherche scientifique (F.R.S.-FNRS).

\section{References}

Abdurrahman F. N., Lu J. R., Chun M. et al. 2018, AJ, 156, 100

Baranec C., Riddle R., Law N. M. et al. 2014, ApJ, 790, L8

Baranec C., Riddle R., Law N. M. 2017, arXiv 1709.07103

Baranec C., Chun M., Hall D. et al. 2018, Adaptive Optics Systems VI, 10703, 1070327

Baug T., Ojha D. K., Gosh S. K. et al. 2018, Journal of Astronomical Instrumentation, 7, 1850003-1881

Close L. M., Males J. R., Morzinski K. M. et al. 2018, Adaptive Optics Systems VI, 10703, 107030L 
Chung H., Ramaprakash A. N., Omar A. et al. 2014, Society of Photo-Optical Instrumentation Engineers (SPIE) Conference Series, 9147, 91470V

Davies R., Kasper M. 2012, ARA\&A, 50, 305

Egner S. E., Masciadri E., McKenna D. 2007, PASP, 119, 669

Fan X., Wang F., Yang J. et al. 2019, ApJ, 870, L11

Finet F., Surdej J. 2016, A\&A, 590, A42

Gaia Collaboration (Prusti, T. et al.) 2016, A\&A, 595, A1

Gaia Collaboration (Brown A. G. A. et al.) 2018, A\&A, 616, A1

Gavel D., Kupke R., Dillon D. et al. 2014, Society of Photo-Optical Instrumentation Engineers (SPIE) Conference Series, 9148, 914805

Georgiev I., Neumayer N., Gässler W. et al. 2019, submitted to MNRAS

Hardy J. W. 1998, Adaptive Optics for Astronomical Telescopes, by John W Hardy, pp. 448. Foreword by John W Hardy. Oxford University Press, Jul 1998. ISBN-10: 0195090195. ISBN-13: 9780195090192, 448

Hart M., Milton N. M., Baranec C. et al. 2010, Natur, 466, 727

Jia P., Basden A., Osborn J. 2018, MNRAS, 479, 829

Jolissaint L., Bouxin A., Gökay U. S. et al. 2018, Adaptive Optics Systems VI, 10703, 1070366

Krone-Martins A., Delchambre L., Wertz O. et al. 2018, A\&A, 616, L11

Law N. M., Morton T., Baranec C. et al. 2014, ApJ, 791, 35

Lemon C. A., Auger M. W., McMahon R. G., Ostrovski F. 2018, MNRAS, 479, 5060

Lu J. R., Chun M., Ammons S. M. et al. 2018, Adaptive Optics Systems VI, 10703, 107030N

LSST Science Collaboration (Abell P. A. et al.) 2009, arXiv:0912.0201

Mackay C., Rebolo-López R., Femenia Castellá B. et al. 2012, Society of Photo-Optical Instrumentation Engineers (SPIE) Conference Series, 8446, 844621

Marchetti E., Hubin N. N., Fedrigo E. et al. 2003, Society of Photo-Optical Instrumentation Engineers (SPIE) Conference Series, 4839, 317

Norris B., Schworer G., Tuthill P. et al. 2015, MNRAS, 447, 2894

Ojha D., Ghosh S. K., Sharma S. et al. 2018, BSRSL, 87, 58

Omar A., Kumar T. S., Krishna Reddy B. et al. 2019, arxiv:1902.05857

Orban de Xivry G., Absil O., Lismont M. et al. 2018, Adaptive Optics Systems VI, 10703, 1070338

Pandey S. B., Yadav R. K. S., Nanjappa N. et al. 2016, BSRSL, 87, 42-57

Rabien S., Angel R., Barl L. et al. 2019, A\&A, 621, A4

Rauer H., Catala C., Aerts C. et al. 2014, ExA, 38, 249

Rigaut F., Minowa Y., Akiyama M. et al. 2018, Adaptive Optics Systems VI, 10703, 1070324

Rigaut F., Neichel B. 2018, ARA\&A, 56, 277

Rusu C. E., Oguri M., Minowa Y. et al. 2016, MNRAS, 458, 2

Sluse D. 2019, BSRSL, 88, 152 (this volume)

Tokovinin A., Baumont S., Vasquez J. 2003, MNRAS, 340, 52

Tokovinin A., Cantarutti R., Tighe R. et al. 2016, PASP, 128, 125003

Ziegler C., Law N. M., Baranec C. et al. 2016, Society of Photo-Optical Instrumentation Engineers (SPIE) Conference Series, 9909, 99095U 